\title{
MASTIGAÇÃO, DEGLUTIÇÃO E SUAS ADAPTAÇÕES NA PARALISIA FACIAL PERIFÉRICA
}

\section{Mastication, deglutition and its adaptations in facial peripheral paralysis}

\author{
Marion Renée Mory ${ }^{(1)}$, Adriana Tessitore ${ }^{(2)}$, LeopoldoNizam Pfeilsticker ${ }^{(3)}$, \\ Euro de Barros Couto Junior ${ }^{(4)}$, Jorge Rizzato Paschoal ${ }^{(5)}$
}

\section{RESUMO}

Objetivo: caracterizar mastigação, fase oral da deglutição e possíveis adaptações funcionais observadas nos portadores de Paralisia Facial Periférica. Método: participaram desta pesquisa 30 indivíduos com Paralisia Facial Periférica grau IV, com história de até 30 dias, sem distinção de etiologia e divididos em três grupos, os que apresentavam a paralisia em até 10 dias, de 11 a 20 e de 20 a 30 dias. As funções mastigação e fase oral da deglutição foram avaliadas tanto com alimento sólido e como com água natural. Os indivíduos responderam questões relacionadas às dificuldades imediatamente após a instalação da paralisia. Os dados foram analisados estatisticamente pelo Teste da Razão de Verossimilhança e pelo Teste Exato de Fisher. Resultados: foram constatadas alterações nas funções de mastigação e fase oral da deglutição pela diminuição do tônus no músculo orbicular dos lábios e do músculo bucinador, que diminuindo a pressão intra-oral, favorece o escape de alimento e líquido. À observação da Fonoaudióloga a variável "derrama líquido enquanto bebe" apresentou dados estatisticamente significante $(p=0,003)$ nos três grupos estudados. A variável "acúmulo de alimento entre os dentes e a gengiva no lado paralisado" foi estatisticamente significante nos grupos de 11 a 20 dias $(p=0,002)$. Conclusão: os indivíduos da amostra mastigam no lado paralisado com dificuldade, mediante ciclos mastigatórios lentos e inconsistentes. Ocorre um incremento nos movimentos de língua para limpeza de resíduos retidos no vestíbulo oral no lado paralisado. Este é o sintoma que mais incomoda o paciente. Apresentam dificuldade no beber de forma contínua. Desenvolvem adaptações para compensar suas dificuldades funcionais.

DESCRITORES: Paralisia Facial; Mastigação; Deglutição; Músculos Faciais; Reabilitação

(1) Fonoaudióloga; Voluntária do Setor de Reabilitação Orofacial do Ambulatório de Paralisia Facial do Hospital de Clínicas da Faculdade de Ciências Médicas da Universidade Estadual de Campinas - UNICAMP; Especialização em Motricidade Oral pelo Centro de Especialização em Fonoaudiologia Clínica - CEFAC

(2) Fonoaudióloga; Coordenadora do Setor de Reabilitação Orofacial do Ambulatório de Paralisia Facial do Hospital de Clínicas da Faculdade de Ciências Médicas da Universidade Estadual de Campinas - UNICAMP; Doutora em Ciências Médicas pela Faculdade de Ciências Médicas da Universidade Estadual de Campinas - UNICAMP - Campinas, São Paulo, Brasil.

(3) Médico Otorrinolaringologista; Cirurgião Crânio-Maxilo-Facial do Ambulatório de Base do Crânio e Paralisia Facial da Disciplina de Otorrinolaringologia do Hospital de Clínicas da Faculdade de Ciências Médicas da Universidade Estadual de Campinas - UNICAMP - Campinas, São Paulo, Brasil.

(4) Estatístico; Assistente Técnico da Prefeitura do Município de São Paulo. Doutorado em Ciências - FMUSP, São Paulo, Brasil.

\section{INTRODUÇÃO}

A interrupção parcial ou total dos impulsos enviados pelo Nervo Facial aos músculos por ele inervados resulta na abolição da mímica facial ${ }^{1-6}$.

Maxila, mandíbula, articulações temporomandibulares, dentes, músculos, ligamentos, língua e lábios formam o Sistema Estomatognático (SE) que, controlado pelo Sistema Nervoso Central, é responsável pela sucção, mastigação, deglutição e fala ${ }^{7}$.

(5) Médico Otorrinolaringologista, Professor Associado da Universidade Estadual de Campinas - UNICAMP; Chefe do Ambulatório da Base do Crânio e na Paralisia Facial na Disciplina de Otorrinolaringologia do Hospital de Clínicas da Faculdade de Ciências Médicas da Universidade Estadual de Campinas - UNICAMP; Doutor em Ciência Médicas pela Universidade Estadual de Campinas - UNICAMP, Campinas, São Paulo, Brasil.

Conflito de interesses: inexistente 
Mastigação é o ato de morder e triturar o alimento transformando-o em partículas menores que, ligadas entre si pela ação misturadora da saliva, constituem o bolo alimentar preparado para ser engolido ${ }^{7,8}$. A função aprendida depende das vias neurais e conexões sinápticas estabelecidas e comandadas pelo córtex cerebral ${ }^{9}$. A mastigação contribui na manutenção do tônus adquirido, e é considerada a função mais importante do SE ${ }^{10,11}$.

O padrão bilateral de mastigação é condição ideal para harmonia funcional dos componentes do SE e é modelo de normalidade ${ }^{3,7,11}$.

Do ponto de vista funcional, a musculatura facial e músculos da mastigação atuam sinergicamente. Durante a mastigação, vários grupos musculares contraem-se coordenadamente, destacando-se, obviamente, os músculos mastigatórios seguidos dos músculos da língua e faciais, especialmente o músculo bucinador e o músculo orbicular dos lábios ${ }^{3,12} \mathrm{~A}$ harmonia morfofuncional do SE pressupõe o equilíbrio entre seus componentes fisiológicos básicos e o desempenho das suas funções com máxima eficiência. Qualquer alteração neuromuscular acarretará perturbação funcional do sistema ${ }^{9,13,14}$.

Nos indivíduos com Paralisia Facial Periférica (PFP), a hemiface paralisada induz à mastigação unilateral, no lado não afetado ${ }^{13,15}$. A contenção alimentar entre as arcadas dentárias está comprometida pela ineficiência do músculo bucinador e pela incompetência labial consequente à flacidez da hemiface afetada ${ }^{3,5,12}$.

Os lábios, desviados para o lado normal e com pouca força de oclusão, determinam diminuição na pressão intra-oral, alterando o equilíbrio entre as estruturas, lábios, bochechas, palato e língua 15-17.

Com o tônus da musculatura orofacial comprometido, haverá escape extra-oral, dificuldade na ingestão de líquidos e comprometimento da função mastigatória ${ }^{12-14,17-20}$.

O paciente desenvolve adaptações individualizadas para alimentar-se ${ }^{13,19}$.

Neste trabalho, levantou-se as características da mastigação, a fase oral da deglutição e as possíveis adaptações funcionais usadas em pacientes com PFP para compensar as limitações que apresentam.

\section{MÉTODO}

Trata de um estudo transversal, de caráter descritivo, desenvolvido no Ambulatório da Base do Crânio e Paralisia Facial do Hospital de Clínicas da Faculdade de Ciências Médicas da Universidade Estadual de Campinas - UNICAMP.
Critérios de inclusão:

- Indivíduos entre 10 e 60 anos com PFP House-Brackmann (HB) IV.

- História de até 30 dias de PFP, sem distinção de etiologia.

- Apresentar todos os dentes em bom estado de conservação.

Critérios de exclusão:

- Pacientes com alterações neurológicas relacionadas à deglutição.

- Falta de elementos dentários.

Foram selecionados os 30 indivíduos que preenchessem os critérios de inclusão, divididos em três grupos segundo o tempo de instalação da paralisia: de 1 a 10 dias, de 11 a 20 dias e de 21 a 30 dias.

Em seguida, examinados pela equipe médica e classificados quanto ao grau de paralisia segundo a escala HB.

Esta pesquisa constou de uma avaliação clínica e um questionário que serviu para que os indivíduos respondessem às questões e para que o examinador observasse as dificuldades funcionais (Figura 1).

O questionário, baseado em queixas anteriormente relatadas por pacientes tratados no Ambulatório de Paralisia Facial do Hospital de Clínicas da Faculdade de Ciências Médicas da Universidade Estadual de Campinas - UNICAMP foi aplicado somente após a nossa avaliação, evitando-se que o paciente fosse influenciado pelas questões e inibisse comportamentos espontâneos durante o exame.

Na avaliação clínica a função de mastigação e a fase oral de deglutição foram filmadas e avaliadas pela fonoaudióloga utilizando-se alimento sólido e água natural. Os pacientes foram orientados a mastigar da forma habitual, como fazem em casa. Não foram orientados nem receberam sugestões durante a avaliação, evitando-se modificar o comportamento habitual.

Utilizou-se o seguinte protocolo:

1. O paciente sentado em cadeira, com os pés totalmente apoiados no chão.

2. Oferta de $1 / 5$ de barra de cereal de $25 \mathrm{~g}$ orientou-se que a comesse como estava habituado.

3. Repetição do procedimento.

4. Em seguida oferta de um copo com água natural a ser tomada da maneira habitual.

5. Avaliação do material filmado com foco nas dificuldades encontradas e nos comportamentos compensatórios.

Em seguida, aplicação do questionário contendo questões relacionadas às dificuldades encontradas pelo paciente para alimentar-se em casa e no momento da avaliação clínica. 


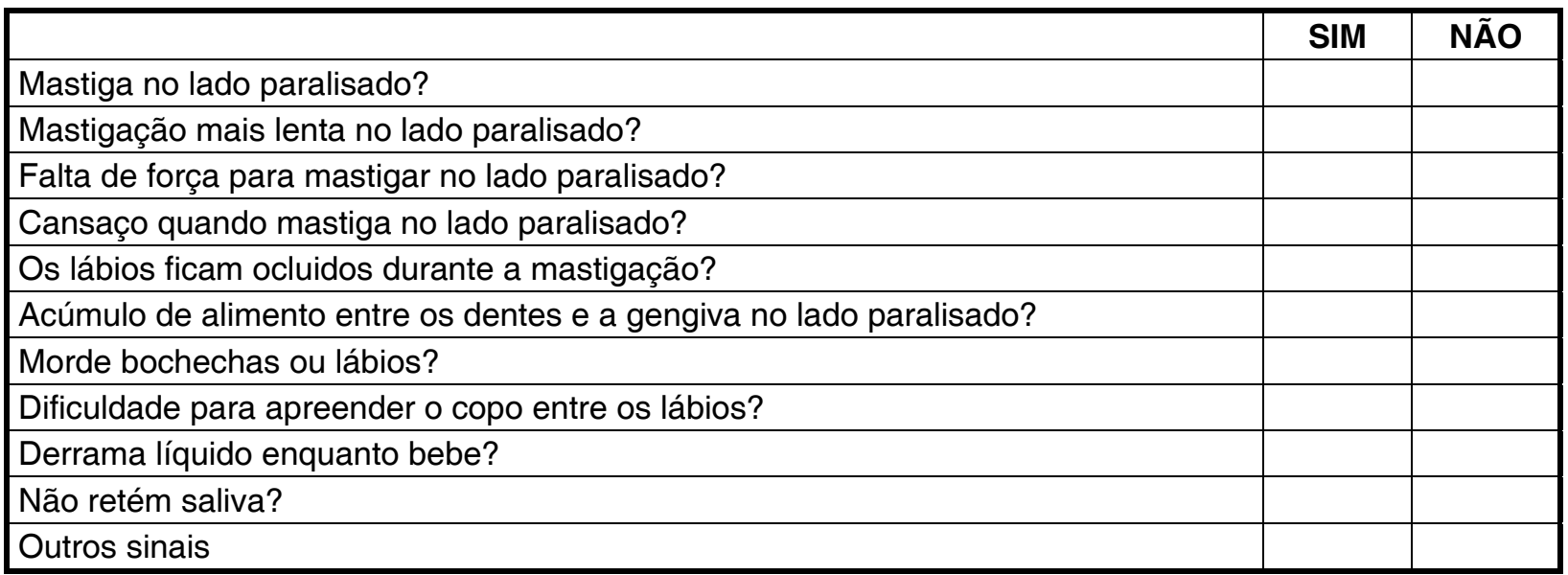

Figura 1 - Protocolo aplicado nos indivíduos portadores de PFP referentes às dificuldades funcionais encontradas para alimentar-se em casa e na avaliação clínica

Essa pesquisa foi avaliada e aprovada pelo Comitê de Ética em Pesquisa da Faculdade de Ciências Médicas da Unicamp com o № 291/2007 tendo sido considerada sem risco e com necessidade de consentimento livre e informado.

Foi aplicado o Teste da Razão de VerossimiIhança, com o intuito de verificar possíveis diferenças entre os três grupos, quando comparados concomitantemente, para as variáveis de interesse:

O nível de significância foi de 5\% (0,050), para a aplicação dos testes estatísticos, ou seja, quando o valor da significância calculada ( $p$ ) sendo menor do que $5 \%(0,050)$, observou-se uma diferença ou uma relação dita estatisticamente significante. Foi aplicado do Teste Exato de Fisher, com o intuito de verificar o grau de associação entre sujeitos e fonoaudióloga, para cada variável de interesse. 0 valor da significância calculada (p) sendo igual ou maior do que $5 \%(0,050)$, representa diferença ou relação 'estatisticamente não-significante'. Assim, neste caso, diferenças entre sujeito e fonoaudióloga serão encontradas, quando $p>0,050$.

\section{RESULTADOS}

A caracterização da amostra contendo o tempo de instalação da PFP, idade, gênero e etiologia dos indivíduos estudados estão apresentadas na Tabela 1.

A distribuição da frequência com seus respectivos percentuais das alterações funcionais relatadas pelos indivíduos com PFP dos três grupos estão dispostos na Tabela 2. Comparados os três grupos, observou-se que existem alterações, mas de acordo com o teste da Razão de Verossimilhança, o tempo não é fator relevante, pois em relação ao mesmo as alterações permanecem.

A distribuição da frequência com seus respectivos percentuais das alterações funcionais observadas pela fonoaudióloga em indivíduos com PFP dos três grupos enquanto se alimentavam, estão dispostas na Tabela 3. De acordo com o teste da Razão de Verossimilhança, houve alteração estatisticamente significante na variável "derrama líquido enquanto bebe" entre os grupos de 11 a 20 dias e 21 a 30 dias na comparação da observação da fonoaudióloga dos três grupos estudados, em que os indivíduos do último grupo apresentaram maior dificuldade neste item.

$\mathrm{Na}$ comparação entre a resposta dos sujeitos por grupo e na observação da fonoaudióloga, houve uma diferença estatisticamente significante na variável "Acúmulo de alimento entre os dentes e a gengiva no lado paralisado" para o grupo de 11 a 20 dias, que foi observado tanto pela fonoaudióloga quanto pelos sujeitos.

$\mathrm{Na}$ comparação entre as alterações funcionais referidas pelos indivíduos com PFP e a observação da fonoaudióloga por grupo, dispostas na Tabela 4, não houve relação estatisticamente significante nas seguintes variáveis:

Para o grupo de 11 a 20 dias, "mastiga no lado paralisado", "dificuldade para apreender o copo entre os lábios" "e "desvio dos lábios para o lado paralisado".

Para o grupo de 21 a 30 dias, "acúmulo de alimento entre os dentes e a gengiva no lado paralisado" e "não retém saliva". 
Tabela 1 - caracterização da população estudada segundo gênero, idade e etiologia

\begin{tabular}{|c|c|c|c|}
\hline $\begin{array}{l}\text { Tempo de } \\
\text { Instalação da PFP }\end{array}$ & Idade (em anos) & gênero & etiologia \\
\hline \multirow{10}{*}{01 a $10 d$} & 11 & $\mathrm{~F}$ & BELL \\
\hline & 36 & $\mathrm{~F}$ & BELL \\
\hline & 54 & $\mathrm{~F}$ & BELL \\
\hline & 60 & $M$ & BELL \\
\hline & 19 & $M$ & TRAUMA \\
\hline & 23 & $\mathrm{~F}$ & BELL \\
\hline & 10 & $\mathrm{~F}$ & BELL \\
\hline & 28 & $M$ & BELL \\
\hline & 30 & $M$ & BELL \\
\hline & 34 & $M$ & BELL \\
\hline \multirow{10}{*}{11 a $20 d$} & 56 & $\mathrm{~F}$ & BELL \\
\hline & 10 & $\mathrm{~F}$ & BELL \\
\hline & 11 & $M$ & BELL \\
\hline & 60 & $\mathrm{~F}$ & BELL \\
\hline & 13 & $\mathrm{~F}$ & BELL \\
\hline & 45 & $\mathrm{~F}$ & BELL \\
\hline & 34 & $\mathrm{~F}$ & TU \\
\hline & 45 & $\mathrm{~F}$ & ZOSTER \\
\hline & 58 & $M$ & TU \\
\hline & 31 & $\mathrm{~F}$ & ZOSTER \\
\hline \multirow{10}{*}{21 a $30 d$} & 60 & $\mathrm{~F}$ & BELL \\
\hline & 33 & $M$ & TRAUMA \\
\hline & 29 & $\mathrm{~F}$ & BELL \\
\hline & 28 & $M$ & $\mathrm{TU}$ \\
\hline & 13 & $M$ & TRAUMA \\
\hline & 42 & $\mathrm{~F}$ & BELL \\
\hline & 44 & $\mathrm{~F}$ & TU \\
\hline & 17 & $\mathrm{~F}$ & BELL \\
\hline & 22 & $M$ & TRAUMA \\
\hline & 56 & $M$ & TRAUMA \\
\hline
\end{tabular}

Legenda:

d: dias

F: feminino

M: masculino

TU: Tumor extrínseco ao nervo facial 
Tabela 2 - distribuição da frequência (em \%) com seus respectivos percentuais das alterações funcionais relatadas pelos indivíduos com pfp dos três grupos segundo as categorias analisadas

\begin{tabular}{lcccc}
\hline Tempo & $\mathbf{n = 1 0}$ & $\mathbf{n = 1 0}$ & $\mathbf{n = 1 0}$ & \\
\cline { 1 - 4 } Alterações (\%) & $\mathbf{1} \mathbf{~ a ~ 1 0 d}$ & $\mathbf{1 1} \mathbf{~ a ~ 2 0 ~ d ~}$ & $\mathbf{2 1}$ a 30 d & \\
\hline Mastiga no LP & 40 & 50 & 70 & 0,384 \\
Mastigação mais lenta no LP & 80 & 80 & 80 & 0,999 \\
Falta de força para mastigar no LP & 20 & 60 & 40 & 0,178 \\
Cansaço quando mastiga no LP & 50 & 30 & 30 & 0,568 \\
Os lábios ficam ocluídos durante a mastigação & 40 & 50 & 50 & 0,874 \\
Desvio dos lábios para o lado são enquanto mastiga & 50 & 50 & 60 & 0,874 \\
Acúmulo de alimento entre os dentes e a gengiva no LP & 80 & 90 & 70 & 0,523 \\
Morde bochechas ou lábios & 50 & 60 & 40 & 0,669 \\
Dificuldade para apreender o copo entre os lábios & 80 & 70 & 60 & 0,617 \\
Derrama líquido enquanto bebe & 70 & 70 & 70 & 0,999 \\
Não retém a saliva & 20 & 40 & 30 & 0,617 \\
Outros sinais & 20 & 20 & 10 & 0,773 \\
\hline
\end{tabular}

Legenda: PFP: paralisia facial periférica

d: dias

LP: lado paralisado

Teste da Razão de Verossimilhança - Nível de significância de $5 \%(0,050)$.

(p) $<5 \%(0,050)=$ diferença estatisticamente significante.

Tabela 3 - Distribuição da frequência (em \%) com seus respectivos percentuais das alterações funcionais observadas pela fonoaudióloga em indivíduos com PFP dos três grupos enquanto se alimentavam segundo as categorias analisadas

\begin{tabular}{lcccc}
\hline Tempo & $\mathbf{n = 1 0}$ & $\mathbf{n = 1 0}$ & $\mathbf{n = 1 0}$ & \\
\cline { 1 - 4 } Alterações (\%) & $\mathbf{1 ~ a ~ 1 0 d}$ & $\mathbf{1 1} \mathbf{~ a ~ 2 0 ~ d ~}$ & $\mathbf{2 1}$ a 30 d & \\
\hline Mastiga no LP & 60 & 70 & 40 & 0,384 \\
Mastigação mais lenta no LP & 80 & 40 & 40 & 0,104 \\
Falta de força para mastigar no LP & 30 & 10 & 10 & 0,404 \\
Cansaço quando mastiga no LP & 40 & 30 & 10 & 0,270 \\
Os lábios ficam ocluídos durante a mastigação & 10 & 20 & 30 & 0,523 \\
Desvio dos lábios para o lado são enquanto mastiga & 70 & 70 & 60 & 0,862 \\
Acúmulo de alimento entre os dentes e a gengiva no LP & 70 & 90 & 80 & 0,523 \\
Morde bochechas ou lábios & 10 & 10 & 10 & 0,999 \\
Dificuldade para apreender o copo entre os lábios & 90 & 60 & 60 & 0,199 \\
Derrama líquido enquanto bebe & 20 & 00 & 60 & 0,003 \\
Não retém a saliva & 20 & 00 & 20 & 0,170 \\
Outros sinais & 00 & 00 & 00 & 0,999 \\
\hline
\end{tabular}

Legenda: PFP paralisia facial periférica

d: dias

LP: lado paralisado

Teste estatístico: Teste da Razão de Verossimilhança - Nível de significância de 5\% $(0,050)$.

$\mathrm{P}<5 \%(0,050)=$ diferença estatisticamente significante. 
Tabela 4 - Comparação entre alterações funcionais referidas pelos indivíduos com pfp e a observação da fonoaudióloga - estudo por grupo - significância do $p$

\begin{tabular}{lccc}
\hline & $\mathbf{1}$ a 10 d & $\mathbf{1 1}$ a $\mathbf{2 0} \mathbf{~ d ~}$ & $\mathbf{2 1}$ a 30 d \\
\hline Mastiga no lado paralisado & ---- & 0,083 & ---- \\
Acúmulo de alimento entre os dentes a gengiva no lado paralisado & ---- & ---- & 0,067 \\
Dificuldade para apreender o copo entre os lábios & ---- & 0,067 & ---- \\
Não retém a saliva & ---- & ---- & 0,067 \\
Desvio dos lábios para o lado não paralisado enquanto mastiga & ---- & 0,083 & ---- \\
\hline
\end{tabular}

Aplicação do Teste Exato de Fisher, diferenças entre sujeito e fonoaudióloga serão encontradas, quando p > 0,050.

\section{DISCUSSÃO}

Os indivíduos com PFP são encaminhados para Fonoterapia com o objetivo de manter o tônus e facilitar a função da musculatura orofacial.

Entre as queixas apresentadas, as dificuldades relacionadas à alimentação são constantes e variam de um para outro. Na literatura consultada, encontram-se descrições dos sinais e sintomas da PFP, das propostas terapêuticas, mas poucos estudos enfocam as dificuldades para comer e beber.

Neste estudo, observou-se as dificuldades na mastigação, na fase oral da deglutição, e as adaptações desenvolvidas para alimentar-se.

Independentemente da severidade da paralisia, os indivíduos com PFP apresentam muitos problemas funcionais imediatamente após a instalação da mesma ${ }^{19}$.

Observou-se que nos 10 primeiros dias, os indivíduos com PFP, ainda sob o impacto causado pela PFP no comprometimento das expressões faciais, não percebem ou não conseguem precisar com certeza as alterações relacionadas à alimentação. $A$ perda dos movimentos voluntários e do tônus orofacial acarreta dificuldades no processo alimentar ${ }^{12,17}$.

A maioria dos indivíduos avaliados (60\%) não relata espontaneamente mastigar no lado paralisado, referindo que a mastigação ficou mais lenta, com acúmulo de alimento no vestíbulo lateral no lado paralisado. A manutenção do alimento entre as arcadas dentárias está comprometida pela flacidez e falta de participação do músculo bucinador e consequentemente há dificuldade de mastigar no lado comprometido ${ }^{11}$.

$\mathrm{Na}$ avaliação clínica funcional, no mesmo período, foram observados os mesmos sinais relatados acima pelos indivíduos.

A sinergia funcional neuromuscular da musculatura mastigatória, facial, lingual e do palato, necessária na alimentação, desorganiza-se na PFP 9,17. A mastigação é realizada em ciclos lentos e inconsistentes provavelmente por encontrar dificuldade na contração coordenada dos movimentos de mandíbula, bochechas e lábios.

Diferente do relatado pelos indivíduos, na avaliação clínica, $60 \%$ deles mastigaram no lado paralisado, necessitando do apoio manual para retirada de resíduos retidos, associado a aumento dos movimentos de língua para limpar o vestíbulo oral no lado paralisado.

Na fase inicial da PFP, com a musculatura flácida do lado da paralisia, os indivíduos não conseguem ou evitam mastigar neste lado pelo acúmulo de alimento, por uma deficiência de ação do músculo bucinador ${ }^{13}$.

Nos primeiros 10 dias, alguns indivíduos não relatam ou não percebem durante a mastigação os lábios entreabertos e desviados para o lado não paralisado permitindo a visibilizar o bolo alimentar na cavidade oral, como foi observado na avaliação clínica.

A duração e a amplitude dos movimentos dos músculos faciais são influenciadas pelos ciclos mastigatórios e pelo contato dos lábios superior e inferior ${ }^{3}$ As ações dos músculos bucinador e orbicular da boca são fundamentais a eficiência mastigatória. Portanto, nos casos com PFP, a eficiência mastigatória estará comprometida, pela inadequação funcional destes músculos.

Estudos referem que pacientes com PFP apresentam preferência mastigatória pelo lado são. Dados eletromiográficos demonstram que a condição dos orbiculares da boca não é fator influente na preferência mastigatória e, sim, a falta de atividade do músculo bucinador ${ }^{3}$.

Os pacientes relataram dificuldade em apreender o copo entre os lábios, havendo escape de líquido enquanto bebiam. A redução da força na oclusão labial implica na diminuição da pressão intra-oral e, em função disso, retenção de líquidos na cavidade oral ${ }^{2,16,17}$.

$\mathrm{Na}$ avaliação clínica, os indivíduos apoiavam o copo no lábio, no lado intacto da face, sem haver escape de líquido, pois tomavam o líquido pouco a pouco, com intervalo entre as tomadas. Esta já seria 
uma forma de adaptação do paciente em relação à ingestão de líquidos.

Os indivíduos que apresentavam PFP de 11 a 20 dias relataram os mesmos sinais que o grupo precedente, acrescidos de mordidas na parte interna das bochechas durante a mastigação e falta de força para mastigar. Acredita-se que a busca pelo mesmo ritmo mastigatório antecedendo a instalação da PFP aumenta a chance de morder a parte interna da bochecha pela ausência de movimento ou pequena participação do músculo bucinador durante a mastigação.

Estudos eletromiográficos, durante a mastigação, realizados em indivíduos com PFP apontam que não há diferença estatisticamente significante entre os masseteres do lado são e do lado com PFP ${ }^{3}$. Desta maneira, a falta de força parece relacionada à dificuldade de manter o sinergismo entre músculos mastigatórios e músculos da face, o que determina movimentos sincronizados da mandíbula e manutenção do alimento sobre os dentes para a trituração.

Este grupo com PFP de 11 a 20 dias não apresentou diferenças estatisticamente significantes para as variáveis mastigar no lado paralisado, desvio dos lábios, dificuldade de apreensão, coincidindo a percepção dos indivíduos com a observação da fonoaudióloga. Entende-se que por estarem com a face paralisada há mais de 10 dias, identificam com maior precisão as dificuldades encontradas para alimentar-se.

Os indivíduos avaliados que apresentavam PFP de 21 a 30 dias, além dos sintomas citados pelos grupos anteriores, relataram mastigar no lado paralisado, mas com ciclos mastigatórios lentos. Percebem os lábios desviados para o lado não paralisado $(60 \%)$, e que estes não ficam selados durante a mastigação (50\%). Relatam dificuldade para apreender o copo entre os lábios, e escape de líquido enquanto bebem. Este último foi um dado estatisticamente significante na comparação entre os três grupos na avaliação clínica.

Acumular alimento no vestíbulo lateral e não reter saliva não apresentou relação estatisticamente significante, isto é tanto foi relatado pelos pacientes, como observado na avaliação clínica. Neste grupo tinham-se quatro casos de PFP por trauma e quatro por tumor extrínseco ao nervo facial e uma possível diferença de tônus entre estes e os pacientes com PFP de Bell podem ter determinado sinais mais acentuados.

Comparando-se os três grupos em relação ao tempo, observou-se que os sinais e sintomas não se alteram. Portanto, o tempo não é um fator relevante.

Inicialmente incomodados, de maneira progressiva desenvolvem adaptações para poder alimentar-se.

Mesmo com dificuldade os indivíduos com PFP mastigam no lado paralisado e o sinal que mais incomoda é a retenção de alimento no vestíbulo com ou sem mordedura de bochechas. Os indivíduos têm consciência de que comem e bebem mais devagar. Desenvolvem adaptações como diminuir a quantidade de alimento, tomar porções menores, preferir alimentos de consistência pastosa e usam o apoio manual para retirada de resíduos. Bebem apoiando o copo no lado não afetado, tomando aos poucos ou usando canudo.

Como na maioria dos casos de Paralisia Bell ocorre uma recuperação espontânea, seria interessante pesquisarem-se as alterações na musculatura orofacial, nas paralisias decorrentes de outras etiologias. Ao permanecerem período mais longo na fase flácida, e devido à recuperação incompleta em muitos casos, poder-se-ia observar as adaptações mais bem desenvolvidas ao alimentar-se.

Nesse estudo, contou-se com o exame subjetivo do Fonoaudiólogo e com o relato individual de cada portador de PFP. Talvez fosse interessante buscar exames objetivos, como a eletromiografia de superfície, para caracterizar os distúrbios motores orais, nas alterações da mastigação, deglutição e da fala nos portadores de paralisia facial ${ }^{3,17}$.

\section{CONCLUSÃO}

Os indivíduos mastigam no lado paralisado com dificuldade, apresentando ciclos mastigatórios lentos e inconsistentes, associados a aumento dos movimentos de língua para a remoção de resíduos no vestíbulo oral. Sentem dificuldade em beber de forma contínua, e buscam adaptações individualizadas, conscientes ou não, que visam a compensar suas dificuldades funcionais. 


\section{ABSTRACT}

Purpose: to describe mastication, swallowing oral phase and possible functional adaptations observed in Facial Peripheral Paralysis subjects. Method: there were 30 subjects with grade IV Facial Peripheral Paralysis, with at the most 30 days paralysis history and no etiology differentiation. They were separated in three groups, 1 to 10 days paralysis, 11 to 20 days and 21 to 30 days. Mastication and swallowing oral phase functions were assessed with both solid food and water. Participants answered questions related to the difficulties right after the paralysis. Data were statistically analyzed using the Likelihood Ratio Test and Fisher Exact Test. Results: changes were observed in mastication and swallowing oral phase due to the lowering of lips tonus, orbicular muscle and buccinator muscle that allows the escaping of food and liquid by decreasing intra-oral pressure. To the speech therapist's observation "spill liquid while drinking" presented statistically significant data $(p=0,003)$ in the three observed groups. Variable "accumulate food between teeth and gums" was statistically significant in groups of 11 to 20 days ( $p=0,002)$. Conclusion: sample subjects chew with difficulty in the paralyzed side, showing slow and inconsistent mastication cycles. There is an increased tongue movements for cleaning the residues kept in oral vestibule in the paralyzed side. This is the most annoying symptom according to the patients. They develop adaptation strategies to compensate their functional difficulties.

KEYWORDS: Facial Palsy; Mastication; Deglutition; Facial Muscles; Rehabilitation

\section{REFERÊNCIAS}

1. Macedo FJM, Konig BJr, Chadi G. - Estudo do desenvolvimento do tubérculo articular da articulação temporomandibular em embriões humanos. Ortop Rev Int Ortop Func. 2004;1(1) 7-24. 2. Dib GC; Kosugi EM, Antunes ML. Paralisia facial periférica Como Diagnosticar e tratar. Rev.Bras. Méd. 2004;61(3):110-7.

3. Carvalho ARR. Preferência mastigatória em pacientes com paralisia facial periférica flácida de duração igual ou superior a seis meses: estudo clínico e eletromiográfico [tese]. São Paulo (SP): Faculdade de Medicina, Universidade de São Paulo; 2008.

4. Bento RF, Tsuji RK. Paralisia facial. In: R Figueiredo (Org). Urgências em otorrinolaringologia. Rio de Janeiro (RJ): Revinter; 2006.p.129-44.

5. Magalhães Junior, Hipólito Virgílio. Fonoterapia na Paralisia Facial Periférica - Uma abordagem Miofuncional- Orofacial Rev. Bras. em Promoção da Saúde. out-dez 2009;22(4):259-63.

6. Veillon F, Taboada LR, Eid MA; Riehm S, Derby $C$, Charpiot A. Pathology of the facial nerve. Neuroimaging Clin N Am. 2008;18(2):309-20.

7. Douglas CR.- Fisiologia da mastigação. In: Fisiologia aplicada à Fonoaudiologia. Rio de Janeiro (RJ): Guanabara - Koogan; 2006. p.325-50.

8. Pereira LJ, Duarte Gavião MB, Van Der Bilt A. Influence of oral characteristics and food products on masticatory function. Acta Odontol Scand. 2006; 64(4):193-201.
9. Whitaker ME, Trindade Jr AS, Genaro KF. Proposta de avaliação clinicada função mastigatória. Rev. CEFAC. 2009;11(3):311-23.

10. Bento RF, Brito Neto RV. Tratamento das paralisias faciais - presente e futuro. In: Lavinsky $L$ (Org.). Tratamento em otologia. Rio de Janeiro (RJ): Revinter; 2005. p.709-15.

11. oPastanaSG; CostaMS, ChiappettaMLA. Análise da mastigação em indivíduos que apresentam mordida cruzada unilateral na faixa-etária de 07 a 12 anos. Rev CEFAC. 2007;9(3):339-50.

12. Tessitore A, Paschoal JR, Pfeilsticker LN.Avaliação de um protocolo da reabilitação orofacial na Paralisia Facial Periférica. Rev. CEFAC. 2009;11(3):432-40.

13. Rahal A, Goffi-Gomez MVS. Avaliação eletromiográfica do músculo masseter em pessoas com paralisia facial periférica de longa duração. Rev CEFAC. 2007;9(2):207-12.

14. Bernardes DFF; Gomez,MVSG, Bento,RF. Eletromiografia de superfície em pacientes portadores de paralisia facial periférica. Rev CEFAC. 2010;12(1):91-6.

15. Queiroz R, Testa JRG, Tessitore A, Paschoal JR. Reabilitação Neuro oclusal e ortopedia funcional dos maxilares em paciente com paralisia facial periférica - relato de caso. ACTA ORL Técnicas em Otorrinolaringologia. 2007;25(4):312-7.

16. Tessitore A, Pfeilsticker LN, Paschoal JR. Aspectos neurofisiológicos da musculatura facial visando a reabilitação na paralisia facial. Rev. CEFAC.2008;10(1):68-75. 
17. Tessitore A. Medida angular para determinação do tônus muscular na paralisia facial. [tese]. Campinas: Faculdade de Ciências Médica, Universidade Estadual de Campinas; 2010.

18. Quintal M, Tessitore A, Pfeilsticker LN,Paschoal JR. Quantificação da Paralisia Facial com paquímetro digital. Rev CEFAC. 2004;6(2):170-6.
19. de Swart BJ, Verheij JC, Beurskens $\mathrm{CH}$. Problems with eating and drinking in patients with unilateral peripheral facial paralysis. Dysphagia. 2003;18(4):267-73.

20. Solomon NP. What is orofacial fatigue and how does it affect function for swallowing and speech? Semin Speech Lang.2006;27(4):268-82.

http://dx.doi.org/10.1590/S1516-18462012005000076

RECEBIDO EM: 09/09/2011

ACEITO EM: 07/12/2011

Endereço para correspondência:

Marion Renée Mory

Rua Nilce Cottini Lombello no 60 casa 9

Jardim das Palmeiras

Campinas - SP

CEP: 13092-596

E-mail: marionfono@terra.com.br

Rev. CEFAC. 2013 Mar-Abr; 15(2):402-410 